\title{
IDENTIFICATION OF RISK CONDITIONS FOR THE DEVELOPMENT OF ADRENAL DISORDERS: HOW OPTIMIZED PUBMED SEARCH STRATEGIES MAKES THE DIFFERENCE
}

\section{This is the author's manuscript}

Original Citation:

Availability:

This version is available http://hdl.handle.net/2318/152520

since 2016-07-27T15:38:48Z

Published version:

DOI:10.1007/s12020-014-0295-x

Terms of use:

Open Access

Anyone can freely access the full text of works made available as "Open Access". Works made available under a Creative Commons license can be used according to the terms and conditions of said license. Use of all other works requires consent of the right holder (author or publisher) if not exempted from copyright protection by the applicable law. 
This is the author's final version of the contribution published as:

F.Guaraldi; M. Parasiliti-Caprino; R. Goggi; G. Beccuti; S. Grottoli; E. Arvat; L. Ghizzoni; E. Ghigo; R. Giordano; D. Gori. IDENTIFICATION OF RISK CONDITIONS FOR THE DEVELOPMENT OF ADRENAL DISORDERS: HOW OPTIMIZED PUBMED SEARCH STRATEGIES MAKES THE DIFFERENCE. ENDOCRINE. 47 (3) pp: 734-739. DOI: $10.1007 / \mathrm{s} 12020-014-0295-\mathrm{X}$

The publisher's version is available at:

http://link.springer.com/content/pdf/10.1007/s12020-014-0295-X

When citing, please refer to the published version.

Link to this full text:

http://hdl.handle.net/2318/152520 


\title{
IDENTIFICATION OF RISK CONDITIONS FOR THE DEVELOPMENT OF ADRENAL DISORDERS: HOW OPTIMIZED PUBMED SEARCH STRATEGIES MAKES THE DIFFERENCE
}

Federica Guaraldi • Mirko Parasiliti-Caprino • Riccardo Goggi • Guglielmo Beccuti • Silvia Grottoli • Emanuela Arvat • Lucia Ghizzoni • Ezio Ghigo • Roberta Giordano • Davide Gori

F. Guaraldi

e-mail: federica.guaraldi@unito.it

F. Guaraldi, M. Parasiliti-Caprino, G. Beccuti, S. Grottoli, L. Ghizzoni, E. Ghigo, R. Giordano Division of Endocrinology, Diabetology and Metabolism, Department of Medical Sciences, University of Turin, Corso Dogliotti, 14, 10126 Turin, Italy

R. Goggi, D. Gori Department of Biomedical and Neuromotor Sciences, School of Hygiene and Preventive Medicine, University of Bologna, Bologna, Italy

E. Arvat Division of Endocrine Oncology, Department of Oncology, University of Turin, Turin, Italy

R. Giordano Department of Clinical and Biological Sciences, University of Turin, Turin, Italy

Key words: Search string $•$ Adrenal diseases $•$ Adrenal disorders $•$ Congenital adrenal disorders $•$ Adrenal genetic diseases

\begin{abstract}
Abstract The exponential growth of scientific literature available through electronic databases (namely PubMed) has increased the chance of finding interesting articles. At the same time, search has become more complicated, time consuming, and at risk of missing important information. Therefore, optimized
\end{abstract}


strategies have to be adopted to maximize searching impact. The aim of this study was to formulate efficient strings to search PubMed for etiologic associations between adrenal disorders (ADs) and other conditions. A comprehensive list of terms identifying endogenous conditions primarily affecting adrenals was compiled. An ad hoc analysis was performed to find the best way to express each term in order to find the highest number of potentially pertinent articles in PubMed. A

predefined number of retrieved abstracts were read to assess their association with ADs' etiology. A more sensitive (providing the largest literature coverage) and a more specific (including only those terms retrieving[ $40 \%$ of potentially pertinent articles) string were formulated. Various researches were performed to assess strings' ability to identify articles of interest in comparison with non-optimized literature searches. We formulated optimized, ready applicable tools for the identification of the literature assessing etiologic associations in the field of ADs using PubMed, and demonstrated the advantages deriving from their application. Detailed description of the methodological process is also provided, so that this work can easily be translated to other fields of practice. 


\section{Introduction}

PubMed (http://www.ncbi.nlm.nih.gov/pubmed/) represents the reference scientific electronic database for answering daily practice questions because of the vastness of the literature sources, free access, and high estimated recall ratio $[1,2]$. MeSH terms (a controlled vocabulary consisting of sets of descriptors arranged in hierarchical structure) and some other search tools to improve search efficacy are provided $[3,4]$. However, methodological limitations in indexing make search complicated, time consuming, and at risk of missing important articles especially when etiological associations are investigated, and the quality of papers is scarce [5]. Most common sources of errors are:

a) MeSH terms assignment: National Library of Medicine librarians ("indexers") arbitrarily choose 5-15 words that best describe article's topic and other main features (i.e., humans, review). They can also assign subheadings, word categories describing a specific field the paper applies to or investigates, i.e., genetics, diagnosis, or therapy). The availability of several similar words makes indexing in each field very heterogeneous. Moreover, MeSH terms are not immediately available as indexing takes from some weeks to more than one year (depending on the journal's impact), so that most recent articles could be missed if MeSH terms only are used in the search strategy.

b) Use of similar or American vs English terminology can lead to very different search outcomes (Supplementary Fig. 1a, b);

c) indexing typos (i.e., hypophysitis' instead of hypophysitis [6]): in this case, the use of truncated words is fundamental to retrieve articles otherwise not identifiable entering the correct word spelling (i.e., hypophysit*).

d) mismatches in Authors' name(s) and surname(s): articles are no longer retrievable if searched using the correct surname (i.e., Matteo and Pierluigi vs Bernardi and Badon [7]; Caprino MP vs. ParasilitiCaprino M [8]). 
Therefore, optimized search strategies have to be adopted to maximize the impact of searching in terms of quality and time [9-15]. We recently formulated the first search strings in endocrinology to investigate pituitary disorders [16]. The aim of this study was to provide efficient and readily applicable strings for the identification of etiologic associations between adrenal disorders (ADs) and any other condition of interest. We also give some elucidating examples that demonstrate a clear search improvement deriving from strings' application.

\section{Materials and methods}

Terms selection

Terms defining endogenous (malformations, neoplasms, autoimmune disorders), congenital, acquired, genetically determined, or sporadic conditions, primarily affecting adrenal medulla or cortex and leading to ADs in humans were considered. ADs secondary to diseases with extraadrenal origin, drugs, chemicals, nutrients, physical agents, or procedures, and studies performed in animals without implications for human health were excluded.

Four sources were used: (1) expert knowledge: each author independently compiled a list of terms (single/ multiple words with specific syntax and Boolean operators) he would have entered in PubMed to test the potential association between a given condition and ADs; (2) authoritative literature source: ADs classification provided by Williams Textbook of Endocrinology [17]; (3) Medline MeSH terms $[\mathrm{MH}]$ and supplementary concepts [SC] database; (4) OMIM database (http://www.ncbi.nlm.nih. gov/omim/): among all genetic syndromes retrieved entering “'adrenal AND (syndrome OR tumor OR disorder)', only those in which adrenal involvement was included in the nosography were considered. To identify the most appropriate way to express keywords (all but $\mathrm{MH}$ or SC terms), each term was assessed as free-text, truncated word (using asterisk), and exact phrase (quoted between inverted commas), using both English and American spelling. 


\section{Estimation of pertinent articles}

Each term was subsequently entered in PubMed using "Abstract available" filter (for practical reasons only abstracts were read) and limiting the search to humans and 2012/12/31 as last date, to be sure that all retrieved articles had been appropriately indexed and MeSH terms applied. The number of abstracts to be read had been previously calculated based on the total number of retrieved articles [16]. Using the “Display setting' menu, the total number of articles extracted was divided into a number of pages close to 100 ; to make sure that abstracts from all the considered time period were included, an appropriate number "top-of-the-page”' articles were kept for each page. Because, we aimed to retrieve the literature with good scientific evidence, only case-control studies, cohort studies, and RCTs were eligible, while case reports or case series, expert opinions or ideas, editorials or letters, ecological studies, and descriptive studies were excluded. Two physicians (D.G.; F.G.) (concordance $\mathrm{j}=0.86$, corresponding to almost perfect agreement [18]) independently assessed articles' pertinence (= investigating ADs' etiology or etiologic associations between ADs and other disorders). In case of disagreement, pertinence was adjudicated by a third reader (M.P.C). For each term, we assessed the number of retrieved articles, the percentage of potentially pertinent articles, the estimated number of potentially pertinent articles, the number of additional abstracts retrieved by the search term but not retrieved by the reference string, and the estimated search overlap (for calculation formulas and results see Supplementary Tables 1, 2). Terms retrieving B1 pertinent article were excluded.

\section{Strings formulation and application}

A more sensitive (including all selected terms and providing the largest literature coverage in the field) and a more specific (including only those terms retrieving[40\% of potentially pertinent articles) string were formulated. This cut-off had been previously and arbitrarily chosen based on personal experience, as no gold standard was available [14]. To both of them, the mini-string "NOT (animals [MH] NOT humans $[\mathrm{MH}])$ )' was added to exclude studies focusing only on animals while keeping those translating 
animal models to clinical practice. The ability of the two optimized strings and of a core string (formulated using terms proposed by Authors; see section "Terms selection-expert knowledge') in retrieving articles associated to AEDs' etiology and etiologic associations between ADs and other unrelated conditions was comparatively assessed by: (1) the number of articles to be read to find a pertinent one (NNR number of calculated pertinent articles/total number of retrieved articles); and (2) the "pertinence odds ratio" $(\mathrm{POR}=$ ratio of occurrence of the event "pertinence" between strings). (Fig. 1). All calculations for estimation of pertinence, NNR, and POR were performed using STATA version 11 (STATA Corp., TX, USA).

\section{Results}

A total of $26 \mathrm{MeSH}$ terms, $8 \mathrm{SC}$, and 45 keywords of interest were identified (Supplementary Table 1) evoking 108,089 articles (34,701 by MeSH terms and SC; 73,379 by keywords). A more sensitive and a more specific string were formulated according to previously specified criteria (Table 1), and compared to the core string. When entered in PubMed to identify articles related to ADs' etiology, the core string found the highest number of articles (104,780 vs 89,339 and 6,837 retrieved by the sensitive and specific string, respectively), but missed a lot of other potentially pertinent ones identified by the two optimized strings $(30,745$ and 3,296, respectively). Optimized strings, particularly the more specific one, resulted also significantly more efficient compared to the core string in retrieving literature addressing etiological associations between ADs and other unrelated disorders, as demonstrated by the proportion of pertinent articles retrieved (specific vs. sensitive: OR 2.37, $\mathrm{p}=0.02$; specific vs. core: OR 3.96, $\mathrm{p}=0.0003$; sensitive vs core OR 1.67, $\mathrm{p}=0.002$ ) (Table 2). Finally, comparing the NNRs of " adrenal $[\mathrm{MH}]$ ", and the core string in their ability to retrieve articles focusing on adrenal origin of “'secondary hypertension', we demonstrated that even a single MeSH term can paradoxically be more suitable for etiological search than a non-optimized string (Supplementary Table 2). 


\section{Discussion and conclusions}

The vastness of accessible electronic literature has increased the chance of finding interesting articles, but also made search more complicated, time consuming and at risk of missing important data because of indexing limitations of databases, unknown to most of the users. Indeed, retrieving the highest number of articles using broad keywords does not necessarily mean picking up all the pertinent ones, nor a good search quality. Therefore, time-effective and efficient search strategies have to be adopted to maximize the impact of searching for specific area of investigation. Wepresenttheresultsofthefirstbibliometricstudyaimed at defining efficient strings readily applicable in PubMed for the identification of putative risk conditions for the developmentofADs.Wesuggesttryingthemorespecificstring - able to retrieve a high proportion of potentially pertinent abstracts - first, then the more sensitive, but also time consuming one, if a deepened research is required. We think the quality of search obtained by applying our strings is high as PubMed covers[90 \% of high-quality papers; terms had been carefully selected and their coverage assessed, and NNR is low. Future potential changes in the terminology related to research and reporting practices in this field might affect the strings' comprehensiveness or efficacy. An update every 3-4 years could be, therefore, indicated. In conclusion, the proposed strings can be helpful to the broader medical community to identify putative risk factors for or disorders associated to ADs, or make literature survey in this field. Following the specified steps, this approach can be translated to other medical fields.

Conflict of interest The Authors declare that they have no conflict of interest.

\section{References}


1. J.F. Gehanno, C. Paris, B. Thirion, J.F. Caillard, Assessment of bibliographic databases performance in information retrieval for occupational and environmental toxicology. Occup. Environ. Med. 55, 562$566(1998)$

2. L. Rollin, S. Darmoni, J.F. Caillard, J.F. Gehanno, Searching for high-quality articles about intervention studies in occupational health-what is really missed when using only the Medline database? Scand. J. Work Environ. Health 36, 484-487 (2010)

3. National Library of Medicine (2010) Factsheet PubMed_: MEDLINE_ Retrieval on the World Wide Web. Bethesda (MD): National Library of Medicine (US). Available from: http://www. nlm.nih.gov/pubs/factsheets/pubmed.html. Last accessed 11 Jan 2014

4. National Library of Medicine (2010) Factsheet PubMed_: medical subject headings (MeSH). Bethesda (MD): National Library of Medicine (US). Available from: http://www.nlm.nih.gov/pubs/ factsheets/mesh.html. Last accessed 11 Jan 2014

5. O. Norgaard, J.V. Lazarus, Searching PubMed during a pandemic. PLoS ONE 5, e10039 (2010)

6. A. Bhansali, P. Velayutham, B.D. Radotra, A. Pathak, Idiopathic granulomatous hypophysitis presenting as non-functioning pituitary adenoma: description of six cases and review of literature. Br. J. Neurosurg. 18, 489-494 (2004)

7. B. Matteo, B. Pierluigi, Descriptive survey about causes of illness given by the parents of children with cancer. Eur. J Oncol. Nurs. 12, 134-141 (2008)

8. B. Lucatello, A. Benso, I. Tabaro, E. Capello, M.P. Caprino, L. Marafetti, D. Rossato, S.E. Oleandri, E. Ghigo, M. Maccario, Long-term re-evaluation of primary aldosteronism after medical treatment reveals high proportion of normal mineralocorticoid secretion. Eur. J. Endocrinol. 168, 525-532 (2013) 9. S. Corrao, D. Colomba, C. Argano, R. Calvo, R. Scaglione, G. Licata, Optimized search strategies for detecting scientifically strong studies on treatment through PubMed. Intern. Emerg. Med. 7, 283287 (2012) 
10. R.B. Haynes, C.D. Mulrow, E.J. Huth, D.G. Altman, M.J. Gardner, More informative abstracts revisited. Ann. Intern. Med. 113, 69-76 (1990)

11. J.J. Allison, C.I. Kiefe, N.W. Weissman, J. Carter, R.M. Centor, The art and science of searching MEDLINE to answer clinical questions. Finding the right number of articles. Int. J. Technol. Assess. Health Care 15, 281-296 (1999)

12. J. Verbeek, J. Salmi, I. Pasternack, M. Jauhiainen, I. Laamanen, F. Schaafsma, C. Hulshof, F. van Dijk, A search strategy for occupational health interventions studies. Occup. Environ. Med. 62, 682$687(2005)$

13. F. Schaafsma, C. Hulshof, J. Verbeek, J. Bos, H. Dyserinck, F. van Dijk, Developing search strategies in Medline on the occupational origin of diseases. Am. J. Ind. Med. 49, 127-137 (2006)

14. S. Mattioli, F. Zanardi, A. Baldasseroni, F. Schaafsma, R.M. Cooke, G. Mancini, M. Fierro, C. Santangelo, A. Farioli, S. Fucksia, S. Curti, F.S. Violante, J. Verbeek, Search strings for the study of putative occupational determinants of disease. Occup. Environ. Med. 67, 436-443 (2010)

15. S. Mattioli, D. Gori, V. Di Gregori, L. Ricotta, A. Baldasseroni, A. Farioli, F. Zanardi, S. Galletti, C. Colosio, S. Curti, F.S. Violante, PubMed search strings for the study of agricultural workers' diseases. Am. J. Ind. Med. 56, 1473-1481 (2013)

16. F. Guaraldi, S. Grottoli, E. Arvat, S. Mattioli, E. Ghigo, D. Gori, PubMed search strategies for the identification of etiologic associations between hypothalamic-pituitary disorders and other medical conditions. Pituitary. 16, 471-482 (2013)

17. S. Melmed, K.S. Polonsky, P.R. Larsen, H.M. Kronenberg, Williams Textbook of Endocrinology, 12th edn. (Elsevier Saunders, Philadelphia, 2011) 18. G.G. Koch, J.R. Landis, The measurement of observer agreement for categorical data. Biometrics. 33, 159-174 (1977) 


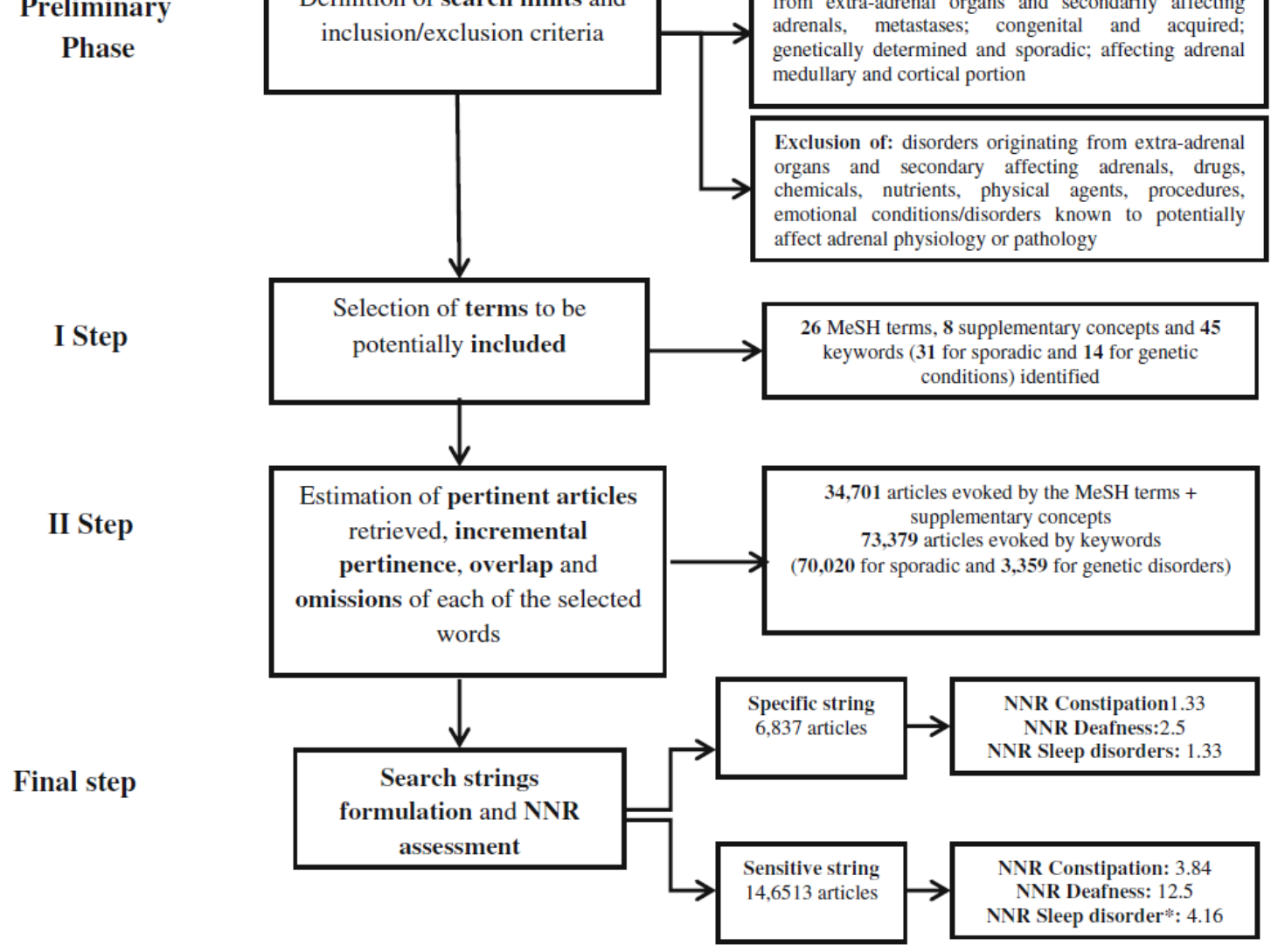

Fig. 1 Methodological flow chart summarizing the main steps followed for strings formulation. Legend NNR number needed to read (number of articles to be read in order to find a pertinent one) 
Table 1 Optimized search strings proposed for the identification of conditions etiologically related to adrenal disorders

\begin{abstract}
Specific string
((18-Hydroxylase deficiency OR Achalasia Addisonianism Alacrimia syndrome OR Addison disease [MH] OR Adrenal hyperplasia, congenital, type 5 OR Autoimmune polyendocrinopathy syndrome, type 1 OR Carney complex [MH] OR Congenital adrenal hyperplasia due to 11-Beta-hydroxylase deficiency OR Congenital adrenal hyperplasia due to 21 hydroxylase deficiency OR Hypoaldosteronism [MH] OR Multiple endocrine neoplasia type 2a [MH] OR Polyendocrinopathies, autoimmune [MH] OR Waterhouse-Friderichsen syndrome [MH] OR X linked adrenal hypoplasia congenita) OR ((“aldosterone producing” adenoma OR "aldosterone producing” adenomas) OR (adrenopaus* OR adrenopause') OR (aldosterone deficiency NOT Hypoaldosteronism [MH]) OR (aldosterone synthase deficiency) OR ("catecholamine secreting" tumor OR "catecholamine secreting" tumors OR "catecholamine secreting" tumor OR "catecholamine secreting" tumors) OR ("cortisol secreting" AND (adenoma OR adenomas)) OR (hypoaldosteronism NOT Hypoaldosteronism [MH])) OR ((Acth-Independent Macronodular Adrenal Hyperplasia) OR (Congenital adrenal hyperplasia due to 17-alpha-hydroxylase deficiency) OR (Congenital adrenal hyperplasia due to cortisone reductase deficiency) OR (Congenital adrenal hyperplasia due to 3-beta-hydroxysteroid dehydrogenase deficiency) OR (Cortisone reductase deficiency 1) OR (Disordered Steroidogenesis due to Cytochrome P450 Oxidoreductase Deficiency) OR (Lipoid Congenital Adrenal Hyperplasia) OR (McCune Albright Syndrome) OR (Primary Pigmented Nodular Adrenocortical Disease OR PPNAD))) NOT (animals [MH] NOT humans [MH]) AND name(s)-of-the-disease
\end{abstract}

\title{
Sensitive string
}

((18-Hydroxylase deficiency OR Achalasia Addisonianism Alacrimia syndrome OR Addison disease [MH] OR Adrenal cortex diseases [MH] OR Adrenal cortex neoplasms [MH] OR Adrenal gland diseases [MH] OR Adrenal gland neoplasms [MH] OR Adrenal hyperplasia 2 OR Adrenal hyperplasia, congenital [MH] OR Adrenal hyperplasia, congenital, type 5 OR Adrenal insufficiency [MH] OR Adrenarche [MH] OR Adrenocortical adenoma [MH] OR Adrenocortical carcinoma [MH] OR Adrenocortical hyperfunction [MH] OR Adrenogenital syndrome $[\mathrm{MH}]$ OR Adrenoleukodystrophy $[\mathrm{MH}]$ OR Autoimmune polyendocrinopathy syndrome, type 1 OR Carney complex $[\mathrm{MH}] \mathrm{OR}$ Congenital adrenal hyperplasia due to 11-Beta-hydroxylase deficiency OR Congenital adrenal hyperplasia due to 21 hydroxylase deficiency OR Cushing syndrome $[\mathrm{MH}]$ OR Hyperaldosteronism $[\mathrm{MH}]$ OR Hyperandrogenism $[\mathrm{MH}]$ OR Hypoaldosteronism [MH] OR

Mineralocorticoid excess syndrome, apparent [MH] OR Multiple endocrine neoplasia type 2a [MH] OR Multiple endocrine neoplasia type $2 \mathrm{~b}[\mathrm{MH}]$ OR Neurofibromatosis 1 [MH] OR Pheochromocytoma [MH] OR Polyendocrinopathies, autoimmune [MH] OR Von hippel-lindau disease $[\mathrm{MH}]$ OR Waterhouse-Friderichsen syndrome $[\mathrm{MH}]$ OR X linked adrenal hypoplasia congenita) OR (((adrenal adenoma OR adrenal adenomas) NOT adrenocortical adenoma $[\mathrm{MH}]$ ) OR ((adrenal carcinoma OR adrenal carcinomas) NOT adrenocortical carcinoma [MH]) OR ((Adrenal disease OR adrenal diseases) NOT "adrenal gland diseases" [MH]) OR ((Adrenal disorder OR adrenal disorders) NOT “adrenal gland diseases"[MH]) OR ((Adrenal neoplasm OR adrenal neoplasms) NOT “adrenal gland neoplasms”[MH]) OR ((Adrenal tumor OR adrenal tumor OR adrenal tumors OR adrenal tumors) NOT “adrenal gland neoplasms" [MH]) OR (adrenal AND (pathology OR pathologies)) NOT epinephrine [MH] OR (adrenal hyperfunction NOT Adrenocortical hyperfunction [mh]) OR (adrenal hypertension) OR adrenalitis OR (adrenal mass OR adrenal masses) OR (adrenopaus* OR adrenopause') OR ("aldosterone producing" adenoma OR "aldosterone producing" adenomas) OR (aldosterone deficiency NOT Hypoaldosteronism [MH]) OR (aldosterone synthase deficiency) OR (ambiguous genitalia NOT "disorders of sex development" [MH]) OR ("androgen secreting" tumor OR "androgen secreting" tumors OR "androgen secreting" tumor OR "androgen secreting" tumors) OR ("catecholamine secreting" tumor OR "catecholamine secreting" tumors OR "catecholamine secreting" tumor OR "catecholamine secreting" tumors) OR ("cortisol secreting" AND (adenoma OR adenomas)) OR ("deoxycorticosterone producing" tumor OR "deoxycorticosterone producing" tumors OR "deoxycorticosterone producing" tumor OR "deoxycorticosterone producing" tumors) OR (electrolytes imbalance) OR (glucocorticoid deficiency) OR (glucocorticoid excess) OR (hyperaldosteronism NOT hyperaldosteronism[MH]) OR (hypercortisolism) OR (hypertensive crisis) OR (hypertrichosis OR hyperthricosi*) OR (hypoadrenalism NOT adrenal insufficiency [MH]) OR (hypoaldosteronism NOT hypoaldosteronism $[\mathrm{MH}]$ ) OR (mineralocorticoid deficiency) OR Precocious adrenarche) OR ((Congenital adrenal hyperplasia due to 17-alpha-hydroxylase deficiency) OR (Congenital adrenal hyperplasia due to cortisone reductase deficiency) OR (Cortisone reductase deficiency 1) OR (Congenital adrenal hyperplasia due to 3-beta-hydroxysteroid dehydrogenase deficiency) OR (17-Beta Hydroxysteroid Dehydrogenase Deficiency) OR (46,XY Sex Reversal 3 OR SRXY3 OR (Congenital Adrenal Insufficiency AND 46, XY Sex Reversal)) OR (Acth-Independent Macronodular Adrenal Hyperplasia) OR (Disordered Steroidogenesis due to Cytochrome P450 Oxidoreductase Deficiency) OR (Familial Hyperaldosteronism NOT hyperaldosteronism [MH]) OR (Familial Hypersecretion of Adrenal Androgens) OR (Hereditary Adrenocortical Carcinoma NOT hyperaldosteronism [MH]) OR (Li Fraumeni Syndrome 1) OR (Lipoid Congenital Adrenal Hyperplasia) OR (McCune Albright Syndrome) OR (Primary Pigmented Nodular Adrenocortical Disease OR PPNAD) OR (Adrenomyodystrophy OR(Alopecia AND Neurologic Defects AND Endocrinopathy Syndrome) OR Chronic Primary Congenital Adrenocortical Hypofunction OR (Ectodermal Dysplasia AND Adrenal Cysts) OR Familial Hypoadrenocorticism OR (Natural Killer Cell AND Glucocorticoid Deficiency AND DNA Repair Defect)))) NOT (animals [MH] NOT humans [MH]) AND name(s)-of-the-disease

It is possible to "copy and paste" each of the two strings into PubMed (either simple or advanced search) from a.doc file

The name(s)-of-the-disease should be entered without any search tag. For diseases that have more than one name, the various "names-of-thedisease" or synonyms should be entered in brackets, connected by the Boolean OR operator (e.g., ... AND (epicondylitis OR tennis elbow))

To be more specific name(s)-of-the-disease could be entered into inverted commas (e.g., "myocardial infarction"), to be more sensitive words can be truncated (e.g., myocard*) 
Table 2 Comparative assessment of sensitive, specific, and core string in retrieving pertinent literature on etiological associations between adrenal disorders and other, apparently unrelated conditions

\begin{tabular}{|c|c|c|c|c|c|c|c|c|c|c|c|c|}
\hline \multirow[t]{2}{*}{ PubMed query } & \multicolumn{3}{|c|}{ Deafness $(n=14,740)$} & \multicolumn{3}{|c|}{ (Sleep disorder*) $(n=16,349)$} & \multicolumn{3}{|c|}{ Constipation $(n=11,340)$} & \multicolumn{3}{|c|}{ Overall $(n=42,332)$} \\
\hline & $R$ & $P(\%)$ & NNR & $R$ & $P(\%)$ & NNR & $R$ & $P(\%)$ & NNR & $R$ & $P(\%)$ & NNR \\
\hline More specific string & 15 & $6(40)$ & 2,5 & 4 & $3(75)$ & 1,33 & 4 & $3(75)$ & 1,33 & 23 & $12(52)$ & 1,92 \\
\hline More sensitive string & 116 & $14(12)$ & 8,28 & 297 & $71(24)$ & 4,18 & 146 & $38(26)$ & 3,84 & 559 & $123(22)$ & 4,54 \\
\hline Core string* & 118 & $12(10)$ & 9,8 & 183 & $27(15)$ & 6,8 & 87 & $19(22)$ & 4,57 & 441 & $58(13)$ & 7,60 \\
\hline
\end{tabular}

$n$ total number of articles retrieved while searching PubMed using the term itself (with previously defined limits), $P$ number of pertinent articles, $R$ number of retrieved articles

* [(adrenal OR adrenal gland* OR adrenal*) OR (adrenal OR “adrenal disease”) OR (adrenal tumor)] 